\title{
COHERENT constraints after the COHERENT-2020 quenching factor measurement
}

\author{
D. K. Papoulias $\odot^{*}$ \\ AHEP Group, Institut de Física Corpuscular-CSIC/Universitat de València, Parc Científic de Paterna. \\ C/Catedrático José Beltrán, 2 E-46980 Paterna (Valencia), Spain
}

(Received 4 August 2019; accepted 30 November 2020; published 15 December 2020)

\begin{abstract}
Recently, an improved quenching factor (QF) measurement for low-energy nuclear recoils in CsI[Na] has been reported by the COHERENT Collaboration. The new energy-dependent QF is characterized by a reduced systematic uncertainty and leads to a better agreement between the experimental COHERENT data and the Standard Model (SM) expectation. In this work, we report updated constraints on parameters that describe the process of coherent elastic neutrino-nucleus scattering within and beyond the SM, and we also present how the new QF affects their interpretation.
\end{abstract}

DOI: 10.1103/PhysRevD.102.113004

\section{INTRODUCTION}

The first observation of coherent elastic neutrino-nucleus scattering $(\mathrm{CE} \nu \mathrm{NS})$ was made at the COHERENT experiment using a CsI[Na] detector at the Spallation Neutron Source (SNS) [1,2], providing a novel powerful probe for a wide range of low-energy physics searches. This motivated a large number of theoretical studies to analyze the recorded $\mathrm{CE} \nu \mathrm{NS}$ signal for performing precision tests of the Standard Model (SM) [3] and for investigating possible signatures of new physics beyond the SM [4-7]. The subject became of intense interest during the latest period, and a plethora of extensive studies constantly appear covering a wide spectrum of new physics phenomena such as nonstandard interactions (NSIs) [8-14], neutrino electromagnetic properties [15-18], sterile neutrinos [19-21], charge-parity violation [22], and new mediators [23-26]. Nuclear and atomic effects were also explored in Refs. [27-33], which may have direct implications for the neutrino-floor [34-36] and dark matter searches [37,38]. Moreover, from the perspective of experimental physics, several experimental proposals aim to measure $\mathrm{CE} \nu \mathrm{NS}$ at the SNS [39] and at reactor facilities [40-47] (for a review, see Ref. [48]).

Experiments looking for $\mathrm{CE} \nu \mathrm{NS}$ and direct dark matter signals are typically based on accurate measurements of the nuclear response and are aiming to achieve $\mathrm{keV}$ or sub-keV threshold capabilities depending on the nuclear target.

\footnotetext{
*dipapou@ific.uv.es
}

Published by the American Physical Society under the terms of the Creative Commons Attribution 4.0 International license. Further distribution of this work must maintain attribution to the author(s) and the published article's title, journal citation, and DOI. Funded by SCOAP ${ }^{3}$.
In such measurements, most of the nuclear recoil energy is dissipated as heat and ionization, while the recorded energy for the case of scintillator detectors is in reality an electron equivalent energy whose magnitude depends on the so-called quenching factor (QF) [49]. The QF is an energy-dependent quantity that is different for a given isotope, and its calibration involves neutron scattering measurements [50]. Regarding the first observation of $\mathrm{CE} \nu \mathrm{NS}$ at COHERENT with a $14.57 \mathrm{~kg} \mathrm{CsI}[\mathrm{Na}]$ detector, the first theoretical simulations adopted an energyindependent $\mathrm{QF}$ of $8.78 \pm 1.66 \%$ in the search region 5-30 $\mathrm{keV}_{\mathrm{nr}}$ [51]. In this work, we employ the new energy-dependent QF resulted by the COHERENT-2020 campaign [52] from a refined analysis correcting systematic effects of previous measurements, i.e., Chicago-1, Chicago-2, and Duke. ${ }^{1}$

We first show that the new QF measurement leads to a higher consistency between the SM expectation and the experimental data, a result that is in agreement with Ref. [53]. We then revisit various constraints on conventional and exotic parameters describing the CE 2 NS interaction and update their status. In the first stage, we explore the sensitivity to the weak mixing angle and to the average nuclear root-mean-square (rms) radius of CsI assuming purely SM interactions. Afterward, we reexamine the sensitivity of COHERENT to phenomenological parameters in the framework of new physics interaction channels such as vector NSIs, neutrino magnetic moments, and charge radii as well as in simplified scenarios with novel vector- $Z^{\prime}$ and scalar mediators. The new constraints are obtained on the basis of an improved $\chi^{2}$ fit analysis that incorporates the aforementioned quenching factor effects.

\footnotetext{
${ }^{1}$ For the Chicago-3 analysis, see Ref. [53].
} 
We show that the new energy-dependent QF combined with the reduced uncertainty leads to stronger constraints compared to previous studies.

The paper is organized as follows: In Sec. II, we provide all necessary ingredients to accurately simulate the observed $\mathrm{CE} \nu \mathrm{NS}$ signal. In Sec. III, we provide the numerical results of our sensitivity analysis and update the constraints on the parameters describing the studied conventional and exotic physics phenomena. Finally, in Sec. IV we summarize the main outcomes of our work.

\section{SIMULATION OF THE COHERENT CE $\nu$ NS RATE}

During the $\mathrm{CE} \nu \mathrm{NS}$ interaction, a neutrino with energy $E_{\nu}$ scatters off a nuclear target $(A, Z)$ with $Z$ protons and $N=$ $A-Z$ neutrons, which in turn produces a detectable nuclear recoil $T_{A}$. Focusing on the COHERENT experiment, after summing appropriately over the nuclear isotopes $x=\mathrm{Cs}$, I and all incident neutrino flavors $\nu_{\alpha}=\left(\nu_{e}, \nu_{\mu}, \bar{\nu}_{\mu}\right)$, the number of expected $\mathrm{CE} \nu \mathrm{NS}$ events is given by

$$
\begin{aligned}
N_{\text {theor }}= & \sum_{\nu_{\alpha}} \sum_{x=\mathrm{Cs}, \mathrm{I}} N_{\mathrm{targ}}^{x} \int_{T_{\mathrm{th}}}^{T_{A}^{\max }} \int_{E_{\nu}^{\min }}^{E_{\nu}^{\max }} f_{\nu_{\alpha}}\left(E_{\nu}\right) \mathcal{A}\left(T_{A}\right) \\
& \times\left(\frac{d \sigma_{x}}{d T_{A}}\left(E_{\nu}, T_{A}\right)\right)_{\lambda} d E_{\nu} d T_{A}
\end{aligned}
$$

and depends on the differential cross section $\left(d \sigma_{x} / d T_{A}\right)_{\lambda}$ that is relevant in the framework of a neutrino interaction channel $\lambda$ within or beyond the SM. The number of target nuclei contained in the CsI detector with mass $m_{\text {det }}=14.57 \mathrm{~kg}$ is determined by Avogradro's number $N_{A}$ and the stoichiometric ratio $\eta_{\chi}$ through the relation $N_{\text {targ }}^{x}=\frac{m_{\text {det }} \eta_{x}}{\sum_{x} A_{x} \eta_{x}} N_{A}$. The neutrino-energy flux at the SNS consists of a prompt and a delayed beam that is adequately described by the Michel spectrum [54]

$$
\begin{aligned}
& f_{\nu_{\mu}}\left(E_{\nu}\right)=\mathcal{N} \delta\left(E_{\nu}-\frac{m_{\pi}^{2}-m_{\mu}^{2}}{2 m_{\pi}}\right) \quad(\text { prompt }), \\
& f_{\bar{\nu}_{\mu}}\left(E_{\nu}\right)=\mathcal{N} \frac{64 E_{\nu}^{2}}{m_{\mu}^{3}}\left(\frac{3}{4}-\frac{E_{\nu}}{m_{\mu}}\right) \quad \text { (delayed) } \\
& f_{\nu_{e}}\left(E_{\nu}\right)=\mathcal{N} \frac{192 E_{\nu}^{2}}{m_{\mu}^{3}}\left(\frac{1}{2}-\frac{E_{\nu}}{m_{\mu}}\right) \quad \text { (delayed) }
\end{aligned}
$$

normalized to $\mathcal{N}=r N_{\mathrm{POT}} / 4 \pi L^{2}$, where $L=19.3 \mathrm{~m}$ is the detector distance from the SNS source, and $r=0.08$ denotes the number of neutrinos per flavor produced for each proton on target (POT), i.e., $N_{\mathrm{POT}}=1.76 \times 10^{23}$ for a period of 308.1 days. Assuming SM interactions, the differential cross section with respect to the nuclear recoil energy is expressed as [55-57]

$$
\left(\frac{d \sigma}{d T_{A}}\right)_{\mathrm{SM}}=\frac{G_{F}^{2} m_{A}}{\pi}\left(Q_{W}^{V}\right)^{2}\left(1-\frac{m_{A} T_{A}}{2 E_{\nu}^{2}}\right) F^{2}\left(Q^{2}\right),
$$

where $m_{A}$ denotes the nuclear mass and $G_{F}$ the Fermi coupling constant. The vector $Q_{W}^{V}$ weak charge is given by [58]

$$
\begin{aligned}
Q_{W}^{V}= & {\left[2\left(g_{u}^{L}+g_{u}^{R}\right)+\left(g_{d}^{L}+g_{d}^{R}\right)\right] Z } \\
& +\left[\left(g_{u}^{L}+g_{u}^{R}\right)+2\left(g_{d}^{L}+g_{d}^{R}\right)\right] N,
\end{aligned}
$$

while the $P$-handed couplings of $u$ and $d$ quarks to the $Z$ boson take the form

$$
\begin{aligned}
g_{u}^{L} & =\rho_{\nu N}^{N C}\left(\frac{1}{2}-\frac{2}{3} \hat{\kappa}_{\nu N} \hat{s}_{Z}^{2}\right)+\lambda^{u, L}, \\
g_{d}^{L} & =\rho_{\nu N}^{N C}\left(-\frac{1}{2}+\frac{1}{3} \hat{\kappa}_{\nu N} \hat{s}_{Z}^{2}\right)+\lambda^{d, L}, \\
g_{u}^{R} & =\rho_{\nu N}^{N C}\left(-\frac{2}{3} \hat{\kappa}_{\nu N} \hat{s}_{Z}^{2}\right)+\lambda^{u, R}, \\
g_{d}^{R} & =\rho_{\nu N}^{N C}\left(\frac{1}{3} \hat{\kappa}_{\nu N} \hat{s}_{Z}^{2}\right)+\lambda^{d, R}
\end{aligned}
$$

Here, $\hat{s}_{Z}^{2} \equiv \sin ^{2} \theta_{W}=0.2382$ is the weak mixing angle, and $\rho_{\nu N}^{N C}=1.0082, \hat{\kappa}_{\nu N}=0.9972, \lambda^{u, L}=-0.0031, \lambda^{d, L}=$ -0.0025 , and $\lambda^{d, R}=2 \lambda^{u, R}=3.7 \times 10^{-5}$ are the radiative corrections [59]. Because of their tiny contributions to the $\mathrm{CE} \nu \mathrm{NS}$ rate, axial-vector interactions, incoherent interactions, as well as contributions due to the sodium dopant of the $\mathrm{CsI}[\mathrm{Na}]$ detector are neglected.

The main source of theoretical uncertainty in the SM $\mathrm{CE} \nu \mathrm{NS}$ process arises from the nuclear form factor that takes into account the finite nuclear size and depends on the variation of the momentum transfer $Q^{2}=2 m_{A} T_{A}$ [31]. Following the COHERENT Collaboration, in this work we adopt the Klein-Nystrand $(\mathrm{KN})$ form factor parametrized as [60]

$$
F_{\mathrm{KN}}=3 \frac{j_{1}\left(Q R_{A}\right)}{Q R_{A}}\left[1+\left(Q a_{k}\right)^{2}\right]^{-1},
$$

where $a_{k}=0.7 \mathrm{fm}$ is the range of the Yukawa potential (over a Woods-Saxon distribution) in the hard sphere approximation with radius $R_{A}=1.23 \times A^{1 / 3}$. We note that regarding the old $\mathrm{QF}$, slight differences from the corresponding results of Ref. [16] throughout the paper are due to the adoption of the $\mathrm{KN}$ form factor, the different neutrino-energy distribution considered, the different value of the weak mixing angle, as well as the binned $\chi^{2}$ analysis performed here (see below).

For a scintillation-based experiment, the measured quantity is the number of photoelectrons (PEs) denoted here as $n_{\mathrm{PE}}$. To account for this mechanism, the CE $\nu$ NS differential rate in events vs nuclear recoil energy gets converted 
to an equivalent differential rate in events vs electron recoil energy through the application of the QF function $Q_{f}\left(T_{A}\right)$, and that in turn gets converted to a PE spectrum via the light yield $\mathcal{L}_{Y}=13.348 \mathrm{PE} / \mathrm{keV}_{\text {ee }}$ measured for electron recoils as

$$
n_{\mathrm{PE}}=Q_{f}\left(T_{A}\right) \mathcal{L}_{Y} T_{A}
$$

In Eq. (1), the acceptance efficiency of the CsI detector is taken into account, which in terms of the photoelectron content of the signal reads ${ }^{2}$ [2]

$$
\mathcal{A}\left(n_{\mathrm{PE}}\right)=\frac{k_{1}}{1+e^{-k_{2}\left(n_{\mathrm{PE}}-x_{0}\right)}} \Theta\left(n_{\mathrm{PE}}\right),
$$

with $k_{1}=0.6655, k_{2}=0.4942, x_{0}=10.8507$, and the modified Heaviside function

$$
\Theta\left(n_{\mathrm{PE}}\right)= \begin{cases}0, & n_{\mathrm{PE}}<5 \\ 0.5, & 5 \leq n_{\mathrm{PE}}<6, \\ 1, & n_{\mathrm{PE}} \geq 6 .\end{cases}
$$

Up to now, previous analyses adopted the energyindependent QF of $8.78 \pm 1.66 \%$ recommended by the COHERENT Collaboration in Ref. [1] which carried a large uncertainty of $25 \% .{ }^{3}$ In the present work, we consider the new energy-dependent QF which came out of the refined COHERENT-2020 measurement with a reduced uncertainty by about a factor of 4 at 3.6\% (for more details, see Ref. [52]). In agreement with Ref. [52], within the SM the new calculation gives a theoretical value of 158 events as compared to the 174 events corresponding to the old QF. At this point, it is rather important to emphasize that a better agreement is now reached with the 134 events observed in Ref. [1]. The corresponding results are compared in Fig. 1 as a function of the PE bins.

\section{NUMERICAL RESULTS}

In the present study, we perform a sensitivity analysis of the parameter set $\mathcal{S}$ in question (see below) that follows from a $\chi^{2}(\mathcal{S})$ fit that is relevant for the CsI detector at the COHERENT experiment and reads [1]

$$
\begin{aligned}
\chi^{2}(\mathcal{S}) & =\min _{\mathrm{a}_{1}, \mathrm{a}_{2}}\left[\sum_{i=4}^{15}\left(\frac{N_{\text {meas }}^{i}-N_{\text {theor }}^{i}(\mathcal{S})\left[1+\mathrm{a}_{1}\right]-B_{0 n}^{i}\left[1+\mathrm{a}_{2}\right]}{\sqrt{N_{\text {meas }}^{i}+B_{0 n}^{i}+2 B_{s s}^{i}}}\right)^{2}\right. \\
& \left.+\left(\frac{\mathrm{a}_{1}}{\sigma_{\mathrm{a}_{1}}}\right)^{2}+\left(\frac{\mathrm{a}_{2}}{\sigma_{\mathrm{a}_{2}}}\right)^{2}\right] .
\end{aligned}
$$

\footnotetext{
${ }^{2}$ Note that the efficiency function is instrumental and does not depend on the QF.

${ }^{3}$ In reality, the QF uncertainty is $18.9 \%$ leading to an overall uncertainty in $\mathrm{CE} \nu \mathrm{NS}$ rate of $25 \%$ [61]. We however adopt the official values reported in Ref. [1].
}

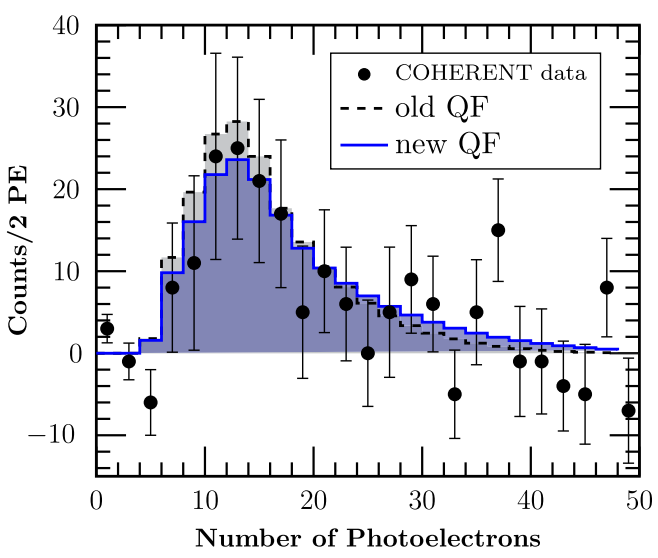

FIG. 1. Comparison of the expected number of events at the COHERENT CsI detector for the old vs the new QF measurement.

Here, $N_{\text {meas }}^{i}\left(N_{\text {theor }}^{i}\right)$ represents the $i$ th bin of the observed signal (theoretical CE $\nu \mathrm{NS}$ events), and $B_{0 n}^{i}\left(B_{s s}^{i}\right)$ denotes the beam-on prompt neutron (steady-state) background events taken from the COHERENT data release [2], while the analysis is restricted to the 12 energy bins corresponding to $6 \leq n_{\mathrm{PE}} \leq 30$. In Eq. (10), $a_{1}$ and $a_{2}$ are the corresponding systematic parameters with fractional uncertainties $\sigma_{\mathrm{a}_{1}}=12.8 \%$ (5\% from signal acceptance determination, $5 \%$ from form factor choice, $10 \%$ from neutrino flux, and 3.6\% from the new QF) and $\sigma_{\mathrm{a}_{2}}=25 \%$. Note that compared to $\sigma_{\mathrm{a}_{1}}=28 \%$ given in Ref. [1] and adopted by all similar studies up to now, the fractional uncertainty considered here is reduced by about a factor of 2. This is also in agreement with estimations of previous studies addressing possible future experimental setups [17,27,31] and will have a direct impact on the updated constraints presented below.

\section{A. SM precision tests and nuclear physics}

Assuming purely SM interactions, we first extract the new sensitivity to the weak mixing angle that arises from the new QF measurement. To this end, we evaluate the $\chi^{2}\left(\sin ^{2} \theta_{W}\right)$ function and perform a sensitivity fit by varying around the central value $\sin ^{2} \theta_{W}=0.2382$. The resultant sensitivity profiles are depicted in the left panel of Fig. 2. A comparison with the corresponding result assuming the old energy-independent QF is also shown. Indeed, this new calculation leads to reasonably improved results. From the fit, we find the following constraints at 90\% C.L.

$$
\begin{aligned}
& \sin ^{2} \theta_{W}=0.197_{-0.080}^{+0.124} \quad(\text { old QF), } \\
& \sin ^{2} \theta_{W}=0.209_{-0.069}^{+0.072} \quad(\text { new QF). }
\end{aligned}
$$

Evaluating the $1 \sigma$ bands $\delta s_{W}^{2}$ according to the definition given in Ref. [15], we find the values $\delta s_{W}^{2}=(0.057,0.042)$ for the (old, new) QF case, which yield the corresponding 

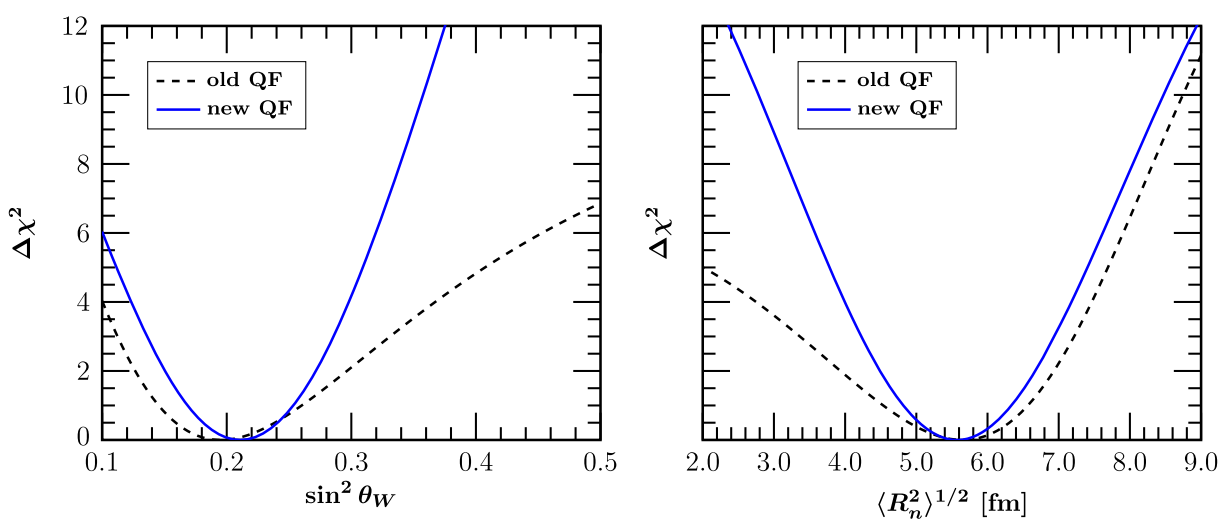

FIG. 2. $\chi^{2}$ profiles for the cases of the weak mixing angle (left) and the average nuclear rms radius of CsI (right) extracted from the analysis of the COHERENT data for the old vs the new QF measurement.

percentage uncertainties $\delta s_{W}^{2} / \sin ^{2} \theta_{W}$ of $(29 \%, 20 \%)$. We then make an effort to explore the sensitivity to the nuclear rms radius that follows from the recent COHERENT measurement. To this purpose, we employ the refined QF resulting from Ref. [52], while in this case we consider the Helm form factor [62]

$$
F_{\text {Helm }}\left(Q^{2}\right)=3 \frac{j_{1}\left(Q R_{0}\right)}{q R_{0}} e^{-(Q s)^{2} / 2},
$$

where $j_{1}(x)$ is the spherical Bessel function of the first kind. Here, $\left\langle R_{n}^{2}\right\rangle^{1 / 2}=\sqrt{\frac{3}{5} R_{0}^{2}+3 s^{2}}$ is the nuclear rms radius, $R_{0}=1.23 A^{1 / 3} \mathrm{fm}$ is the diffraction radius, and $s=0.9 \mathrm{fm}$ quantifies the surface thickness (for more details, see Refs. [30,31]). The resultant sensitivity profile is presented in the right panel of Fig. 2, showing that the constraints are now stronger than previously reported [27,28,31]. In particular, at 90\% C.L. we find the best fits ${ }^{4}$

$$
\begin{aligned}
& \left\langle R_{n}^{2}\right\rangle^{1 / 2}=5.6_{-2.1}^{+1.5} \mathrm{fm} \quad \text { (old QF) }, \\
& \left\langle R_{n}^{2}\right\rangle^{1 / 2}=5.6_{-1.25}^{+1.3} \mathrm{fm} \quad \text { (new QF). }
\end{aligned}
$$

In a similar manner, within $1 \sigma$ error we find the bands $\delta\left\langle R_{n}^{2}\right\rangle^{1 / 2}=(1.01,0.76)$ and the corresponding percentage uncertainties $(18 \%, 14 \%)$ for the (old, new) QF measurement. We finally stress that the latter results remain essentially the same when considering the Klein-Nystrand form factor.

\section{B. Nonstandard interactions}

Nonstandard interactions have been a popular subject of extensive research during the last 15 years, with interesting applications in neutrino oscillations and lowenergy neutrino physics (for a review, see Refs. [63,64]).

\footnotetext{
${ }^{4}$ Note that, in this case the form factor uncertainty is neglected in Eq. (10).
}

For a neutrino with flavor $\alpha=\{e, \mu, \tau\}$ and a quark $q=\{u, d\}$, the vector-type NSI contributions that arise due to nonuniversal (NU) flavor-preserving and flavor-changing interactions are described in the NSI weak charge $[65,66]$

$$
\begin{aligned}
Q_{\mathrm{NSI}}^{V}= & \left(2 \epsilon_{\alpha \alpha}^{u V}+\epsilon_{\alpha \alpha}^{d V}+g_{p}^{V}\right) Z+\left(\epsilon_{\alpha \alpha}^{u V}+2 \epsilon_{\alpha \alpha}^{d V}+g_{n}^{V}\right) N \\
& +\sum_{\alpha, \beta}\left[\left(2 \epsilon_{\alpha \beta}^{u V}+\epsilon_{\alpha \beta}^{d V}\right) Z+\left(\epsilon_{\alpha \beta}^{u V}+2 \epsilon_{\alpha \beta}^{d V}\right) N\right] .
\end{aligned}
$$

In the context of NSI, the expected $\mathrm{CE} \nu \mathrm{NS}$ rate is modified according to the substitution $Q_{W}^{V} \rightarrow Q_{\mathrm{NSI}}^{V}$ in the $\mathrm{SM}$ differential cross section of Eq. (3).

Assuming a single nonvanishing NSI parameter at a time, Fig. 3 illustrates the obtained sensitivity for the NU $\epsilon_{e e}^{q V}\left(\epsilon_{\mu \mu}^{q V}\right)$ couplings in the left (right) panel, while a useful comparison is also given for the case of the old QF. The impact of the new QF measurement on NSI constraints becomes evident. The left and right panels of Fig. 4 show the allowed regions at $90 \%$ C.L. in the $\left(\epsilon_{e e}^{d V}, \epsilon_{e e}^{u V}\right)$ and the $\left(\epsilon_{\mu \mu}^{d V}, \epsilon_{\mu \mu}^{u V}\right)$ parameter space, respectively. We see that the bounds are now more restrictive than the corresponding results using the old $\mathrm{QF}$.

\section{Electromagnetic neutrino interactions}

In this subsection, we are interested in exploring the possibility of probing nontrivial neutrino electromagnetic (EM) properties [67] and to revisit existing constraints from $\mathrm{CE} \nu \mathrm{NS}$ [16]. The two main phenomenological parameters that arise in the framework of EM neutrino interactions are the neutrino magnetic moment and the neutrino charge radius. For completeness, we mention that in the simplest Majorana neutrino case, the neutrino magnetic moment $\mu_{\nu}$ is in reality expressed in terms of the neutrino transition magnetic moments $\Lambda_{i}$ of the neutrino magnetic moment matrix [68,69], while constraints have been recently extracted from neutrino-electron scattering [70] and CELNS [17]. Here, for simplicity, we consider the effective 

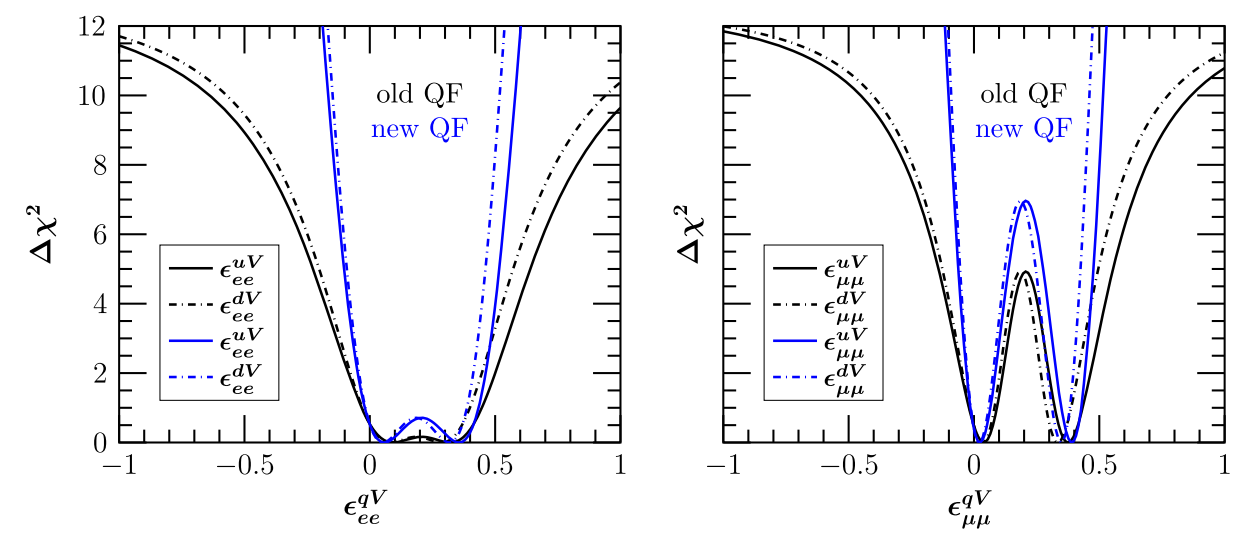

FIG. 3. $\chi^{2}$ profiles for the NU NSIs from the analysis of the COHERENT data. A comparison of the obtained sensitivity using the old vs the new $\mathrm{QF}$ is also shown.
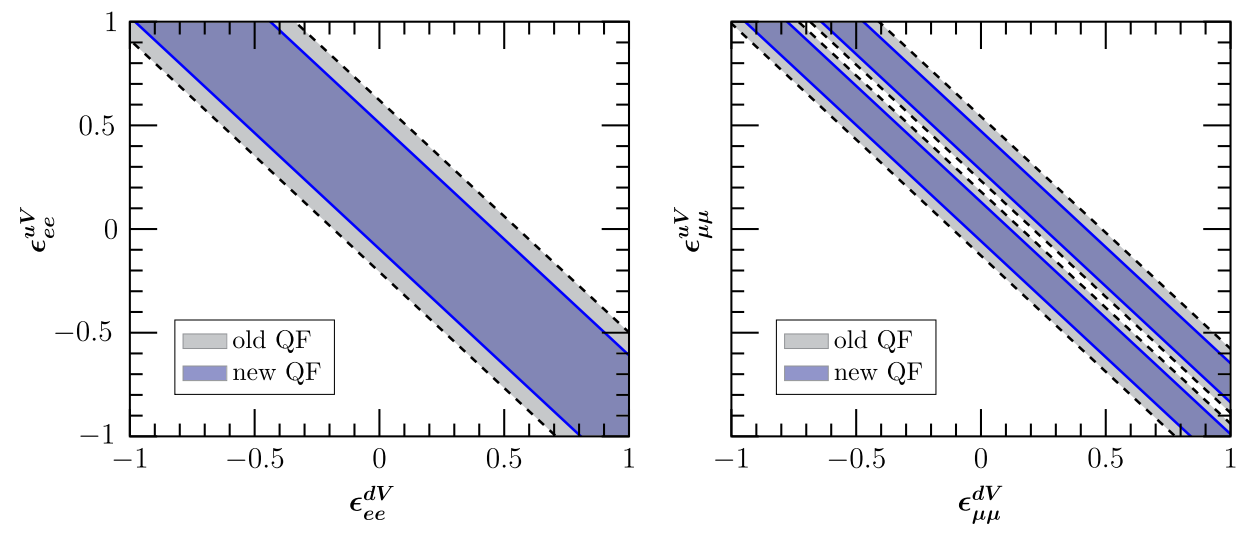

FIG. 4. Allowed regions in the NU NSIs parameter space obtained from the analysis of the COHERENT data for the old vs the new QF measurement.

neutrino magnetic moment in the helicity-violating EM cross section [71]

$$
\left(\frac{d \sigma}{d T_{A}}\right)_{\mathrm{EM}}=\frac{\pi a_{\mathrm{EM}}^{2} \mu_{\nu}^{2} Z^{2}}{m_{e}^{2}}\left(\frac{1-T_{A} / E_{\nu}}{T_{A}}\right) F^{2}\left(Q^{2}\right)
$$

In Fig. 5, we present the updated constraint on $\mu_{\nu}$ from our analysis with the new QF, which is also compared to the corresponding one that comes from the old QF. The obtained upper limits at $90 \%$ C.L. read

$$
\begin{array}{ll}
\mu_{\nu}<3.1 \times 10^{-9} \mu_{B} & (\text { old QF }) \\
\mu_{\nu}<2.6 \times 10^{-9} \mu_{B} & \text { (new QF) }
\end{array}
$$

From the same plot, it can be deduced that this difference is more pronounced at $99 \%$ C.L.

For a flavor neutrino $\nu_{\alpha}$, the associated neutrino charge radius $\left\langle r_{\nu_{\alpha}}^{2}\right\rangle$ is another interesting phenomenological quantity which arises from the helicity-conserving charge form factor of the EM neutrino current [72]. The latter leads to a shift of the weak mixing angle as follows [73]:

$$
\sin ^{2} \theta_{W} \rightarrow \sin ^{2} \overline{\theta_{W}}+\frac{\sqrt{2} \pi a_{\mathrm{EM}}}{3 G_{F}}\left\langle r_{\nu_{\alpha}}^{2}\right\rangle .
$$

We stress that there is not a sign flip regarding antineutrino charge radii; e.g., it holds $\left\langle r_{\bar{\nu}_{\alpha}}^{2}\right\rangle=\left\langle r_{\nu_{\alpha}}^{2}\right\rangle$ as defined in

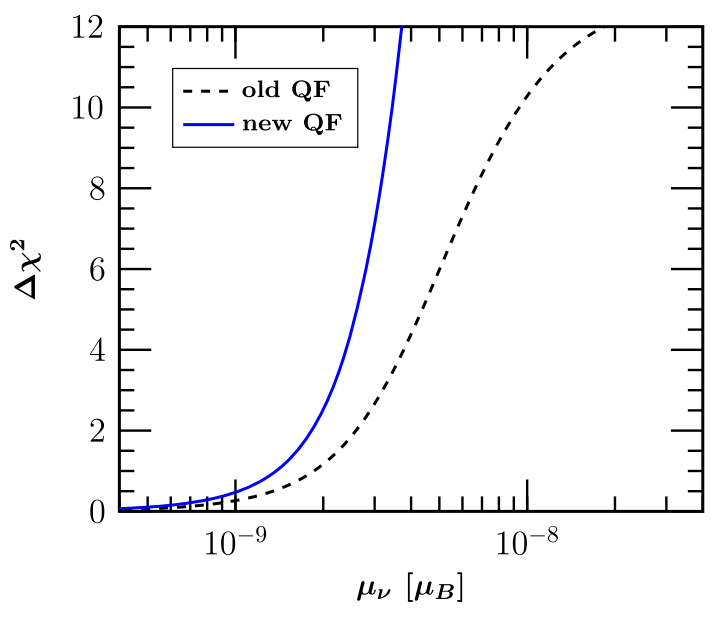

FIG. 5. $\chi^{2}$ profiles of the effective neutrino magnetic moment extracted by the COHERENT data for the old vs the new QF measurement. 


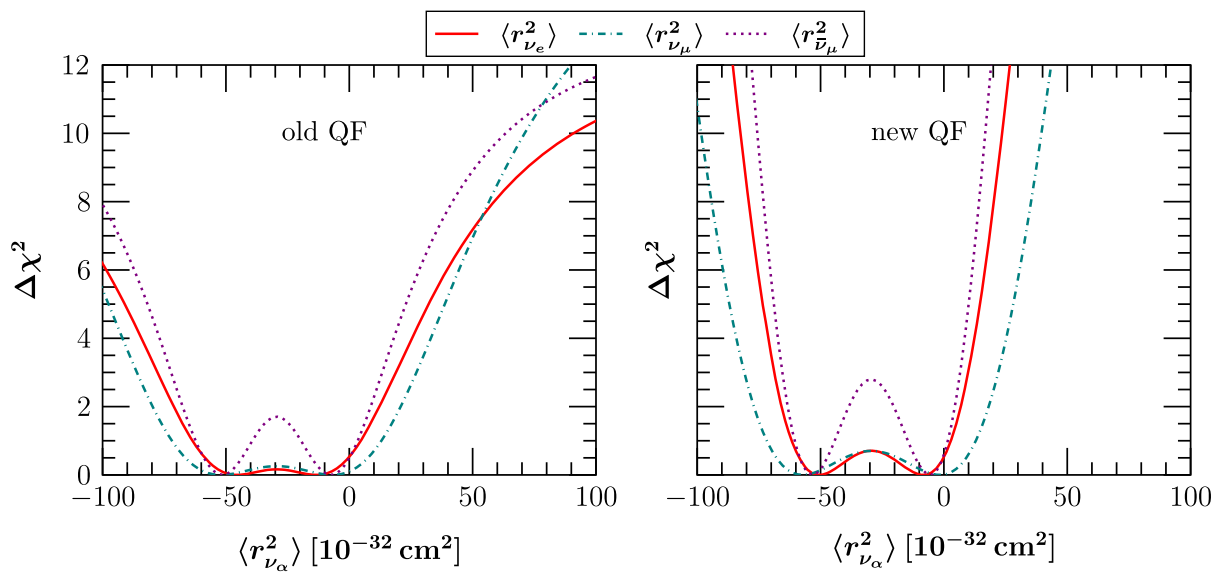

FIG. 6. $\chi^{2}$ profiles of the relevant neutrino charge radii at the COHERENT experiment for the old (left) and the new (right) QF measurement.
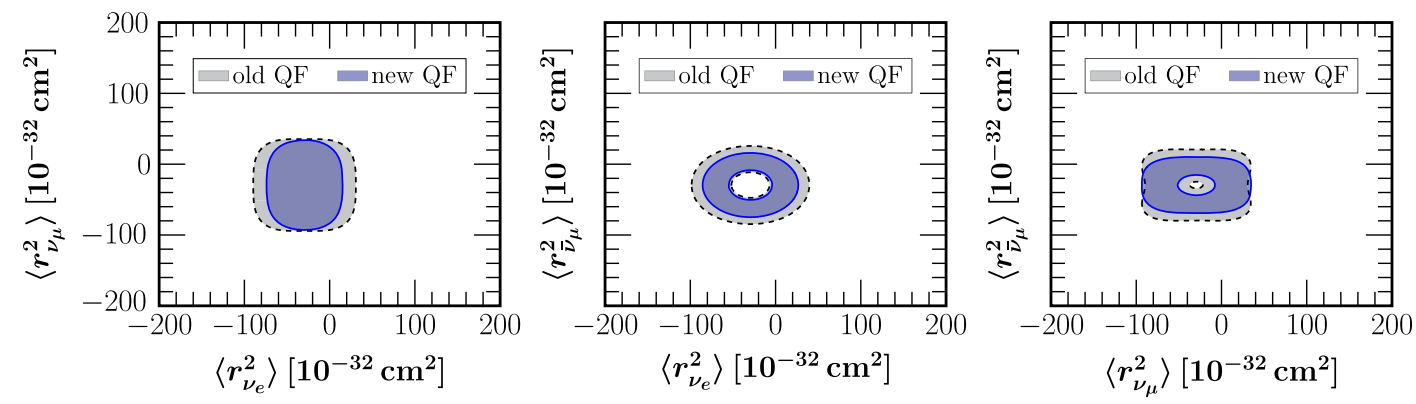

FIG. 7. Contours in the neutrino charge radius parameter space from the analysis of the COHERENT data. The results are shown for various combinations in the $\left(\left\langle r_{\nu_{\alpha}}^{2}\right\rangle,\left\langle r_{\nu_{\beta}}^{2}\right\rangle\right)$ plane and compared for the old vs the new QF measurement.

Ref. [16]. ${ }^{5}$ In this work, we follow the definition given in Ref. [16]; however, the shift considered here is smaller by a factor of 2. Neglecting transition charge radii and varying one parameter at a time, Fig. 6 shows the $\chi^{2}$ profiles of the neutrino charge radii $\left\langle r_{\nu_{\alpha}}^{2}\right\rangle$ associated with the respective SNS neutrino flux, where the left (right) panels correspond to the old (new) QF measurement. The obtained constraints differ slightly due to the old vs new QF data. The only noticeable difference is that by employing the new QF in the case of the prompt $\bar{\nu}_{\mu}$ beam, the resultant constraint on $\left\langle r_{\bar{\nu}_{\mu}}^{2}\right\rangle$ is separated into two distinct regions at $90 \%$ C.L. It is now worthwhile to explore the simultaneous constraints that can be obtained. Figure 7 presents the allowed regions at $90 \%$ C.L. in the $\left(\left\langle r_{\nu_{\alpha}}^{2}\right\rangle,\left\langle r_{\nu_{\beta}}^{2}\right\rangle\right)$ parameter space. As expected, the allowed parameter space in all cases is more restricted using the new QF data.

\footnotetext{
${ }^{5}$ Reference [74] used a negative sign which is now corrected in Ref. [75].
}

\section{Simplified scenarios with light mediators}

In addition to the NSIs discussed previously in Sec. III B, we are now interested in simplified scenarios where the NSI is generated due to the presence of novel mediators.

In the first step, we explore the case where the CE $2 \mathrm{NS}$ rate is enhanced from contributions due to a vector- $Z^{\prime}$ mediator with mass $M_{Z^{\prime}}$. The relevant cross section takes the form [76]

$$
\left(\frac{d \sigma}{d T_{A}}\right)_{\mathrm{SM}+Z^{\prime}}=\mathcal{G}_{Z^{\prime}}^{2}\left(T_{A}, g_{Z^{\prime}}, M_{Z^{\prime}}\right)\left(\frac{d \sigma}{d T_{A}}\right)_{\mathrm{SM}},
$$

with the $Z^{\prime}$ factor defined as

$$
\mathcal{G}_{Z^{\prime}}=1+\frac{1}{\sqrt{2} G_{F}} \frac{Q_{Z^{\prime}}}{Q_{W}^{V}} \frac{g_{Z^{\prime}}^{\nu V}}{2 m_{A} T_{A}+M_{Z^{\prime}}^{2}} .
$$

In the above expression, in order to reduce the number of model parameters, we consider the generalized coupling $g_{Z^{\prime}}^{2}=g_{Z^{\prime}}^{\nu V} Q_{Z^{\prime}} / 3 A$ that is expressed in terms of the vector $\nu_{\alpha}-Z^{\prime}$ coupling times the respective vector charge $Q_{Z^{\prime}}^{V}$, 

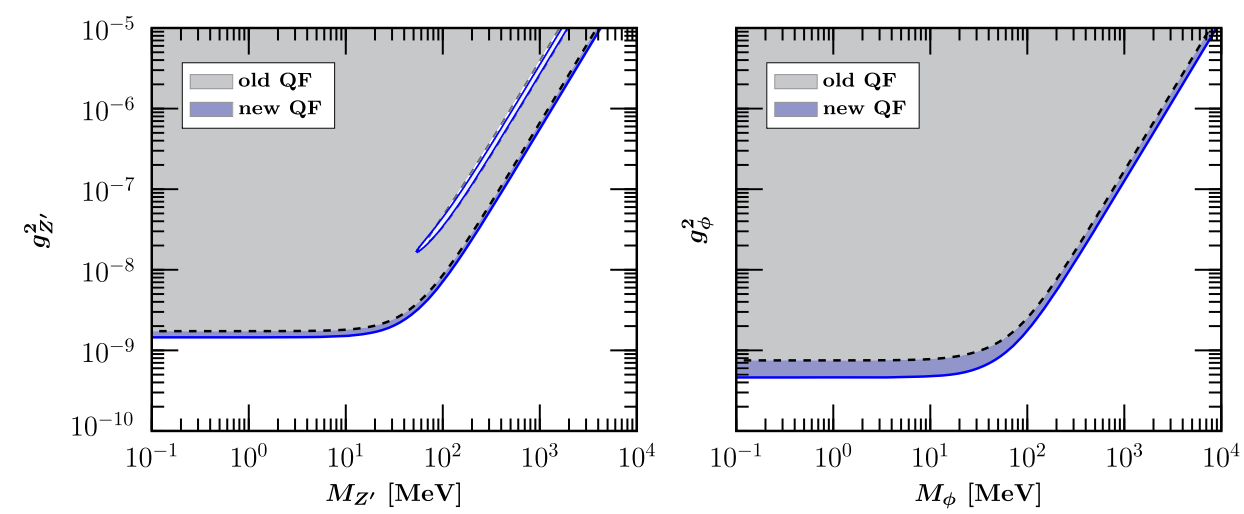

FIG. 8. Exclusion curves in the $\left(g_{Z^{\prime}}^{2}, M_{Z^{\prime}}\right)$ parameter space (left) and in the $\left(g_{\phi}^{2}, M_{\phi}\right)$ parameter space (right) from the analysis of the COHERENT data. The results are shown for the old and the new QF measurement.

under the assumption of universal quark- $Z^{\prime}$ couplings (for more details, see Ref. [16]).

Concentrating our attention on the case of a new scalar boson $\phi$ mediating the $\mathrm{CE} \nu \mathrm{NS}$ process, the cross section takes the form [77]

$$
\left(\frac{d \sigma}{d T_{A}}\right)_{\text {scalar }}=\frac{G_{F}^{2} m_{A}^{2}}{4 \pi} \frac{\mathcal{G}_{\phi}^{2} M_{\phi}^{4} T_{A}}{E_{\nu}^{2}\left(2 m_{A} T_{A}+M_{\phi}^{2}\right)^{2}} F^{2}\left(Q^{2}\right),
$$

with the corresponding scalar factor being

$$
\mathcal{G}_{\phi}=\frac{g_{\phi}^{\nu S} Q_{\phi}}{G_{F} M_{\phi}^{2}} .
$$

In the same spirit of the discussion made above, for the sake of simplification our calculations involve the generalized scalar coupling $g_{\phi}^{2}=g_{\phi}^{\nu S} Q_{\phi} /(14 A+1.1 Z)^{6}$

The exclusion regions in the parameter space $\left(M_{Z^{\prime}}, g_{Z^{\prime}}^{2}\right)$ and $\left(M_{\phi}, g_{\phi}^{2}\right)$ for the vector and scalar scenarios, respectively, are obtained from a two parameter analysis of the COHERENT data. For both old and new QF data, the results are presented at 90\% C.L. in the left (right) panel of Fig. 8 for vector (scalar) mediators. As in all previous cases, from this plot we conclude that the new QF data lead to generally more stringent bounds.

\section{CONCLUSIONS}

Focusing on the COHERENT experiment, we reexamined the results implied from $\mathrm{CE} \nu \mathrm{NS}$ in light of a new QF measurement [52]. By using the new QF data, we came out with improved constraints regarding all the

\footnotetext{
${ }^{6}$ This result derives from the nuclear charge related to the scalar boson exchange; see Ref. [16].
}

cases analyzed in this work. A full summary is given in Table I. At first, we presented updated constraints focusing on important SM parameters, namely, the weak mixing angle and the average nuclear rms radius of CsI, and we explicitly demonstrated the level of improvement. We then concentrated on interesting phenomenological parameters beyond the SM and presented updated constraints for nonuniversal NSIs as well as for electromagnetic neutrino properties including the effective neutrino magnetic moment and the neutrino charge radius. Finally, we revisited the sensitivity of COHERENT in the framework of simplified scenarios involving massive vector and scalar mediators. We concluded that a substantial improvement on SM parameters is reached, while the improvement of beyond the SM physics constraints is also evident.

TABLE I. Summary of constraints at $90 \%$ C.L. in the present work. The results are extracted assuming the old and the new QF data. The nuclear rms radius is in units of $\mathrm{fm}$, the effective neutrino magnetic moment in $10^{-10} \mu_{B}$, and the neutrino charge radius in $10^{-32} \mathrm{~cm}^{2}$.

\begin{tabular}{lcc}
\hline \hline Parameter & Old QF & New QF \\
\hline $\sin ^{2} \theta_{W}$ & $0.116-0.321$ & $0.140-0.282$ \\
$\left\langle R_{n}^{2}\right\rangle^{1 / 2}$ & $3.5-7.1$ & $4.3-6.7$ \\
$\epsilon_{e e}^{u V}$ & $-0.12-0.53$ & $-0.06-0.48$ \\
$\epsilon_{e e}^{d V}$ & $-0.11-0.48$ & $-0.06-0.43$ \\
$\epsilon_{\mu \mu}^{u V}$ & $-0.07-0.13$ & $-0.04-0.1$ \\
& and $0.28-0.49$ & and $0.32-0.45$ \\
$\epsilon_{\mu \mu}^{d V}$ & $-0.06-0.12$ & $-0.03-0.09$ \\
& and $0.25-0.43$ & and $0.28-0.40$ \\
$\mu_{\nu}$ & 30 & 21 \\
$\left\langle r_{\nu_{e}}^{2}\right\rangle$ & $-76-17$ & $-68-9$ \\
$\left\langle r_{\nu_{\mu}}^{2}\right\rangle$ & $-84-25$ & $-80-21$ \\
$\left\langle r_{\bar{\nu}_{\mu}}^{2}\right\rangle$ & $-71-12$ & $-65--32$ and $-27-6$ \\
\hline \hline
\end{tabular}




\section{ACKNOWLEDGMENTS}

The author acknowledges K. Scholberg and J. Collar for useful correspondence. The author is indebted to M. Tórtola and O. Miranda for their critical comments and their help during the preparation of the manuscript.
This work is supported by the Spanish Grants No. SEV2014-0398 and No. FPA2017-85216-P (AEI/FEDER, UE), Grant No. PROMETEO/2018/165 (Generalitat Valenciana), and the Spanish Red Consolider MultiDark Grant No. FPA2017-90566-REDC.
[1] D. Akimov et al. (COHERENT Collaboration), Observation of coherent elastic neutrino-nucleus scattering, Science 357, 1123 (2017).

[2] D. Akimov et al. (COHERENT Collaboration), COHERENT Collaboration data release from the first observation of coherent elastic neutrino-nucleus scattering, https://doi.org/ 10.5281/zenodo.1228631.

[3] B. C. Cañas, E. A. Garcés, O. G. Miranda, and A. Parada, Future perspectives for a weak mixing angle measurement in coherent elastic neutrino nucleus scattering experiments, Phys. Lett. B 784, 159 (2018).

[4] M. Lindner, W. Rodejohann, and X.-J. Xu, Coherent neutrino-nucleus scattering and new neutrino interactions, J. High Energy Phys. 03 (2017) 097.

[5] J. Billard, J. Johnston, and B. J. Kavanagh, Prospects for exploring new physics in coherent elastic neutrino-nucleus scattering, J. Cosmol. Astropart. Phys. 11 (2018) 016.

[6] D. Aristizabal Sierra, V. De Romeri, and N. Rojas, COHERENT analysis of neutrino generalized interactions, Phys. Rev. D 98, 075018 (2018).

[7] O. G. Miranda, G. S. Garcia, and O. Sanders, Testing new physics with future COHERENT experiments, Adv. High Energy Phys. 2019, 3902819 (2019).

[8] J. Liao and D. Marfatia, COHERENT constraints on nonstandard neutrino interactions, Phys. Lett. B 775, 54 (2017).

[9] J. B. Dent, B. Dutta, S. Liao, J. L. Newstead, L. E. Strigari, and J. W. Walker, Accelerator and reactor complementarity in coherent neutrino-nucleus scattering, Phys. Rev. D 97, 035009 (2018).

[10] D. Aristizabal Sierra, N. Rojas, and M. H. G. Tytgat, Neutrino non-standard interactions and dark matter searches with multi-ton scale detectors, J. High Energy Phys. 03 (2018) 197.

[11] P. B. Denton, Y. Farzan, and I. M. Shoemaker, Testing large non-standard neutrino interactions with arbitrary mediator mass after COHERENT data, J. High Energy Phys. 07 (2018) 037.

[12] B. Dutta, S. Liao, S. Sinha, and L. E. Strigari, Searching for Beyond the Standard Model Physics with COHERENT Energy and Timing Data, Phys. Rev. Lett. 123, 061801 (2019).

[13] P. Coloma, M. C. Gonzalez-Garcia, M. Maltoni, and T. Schwetz, COHERENT enlightenment of the neutrino dark side, Phys. Rev. D 96, 115007 (2017).

[14] M. C. Gonzalez-Garcia, M. Maltoni, Y. F. Perez-Gonzalez, and R. Zukanovich Funchal, Neutrino discovery limit of dark matter direct detection experiments in the presence of non-standard interactions, J. High Energy Phys. 07 (2018) 019.

[15] T. S. Kosmas, O. G. Miranda, D. K. Papoulias, M. Tortola, and J.W.F. Valle, Probing neutrino magnetic moments at the Spallation Neutron Source facility, Phys. Rev. D 92 , 013011 (2015).

[16] D. K. Papoulias and T. S. Kosmas, COHERENT constraints to conventional and exotic neutrino physics, Phys. Rev. D 97, 033003 (2018).

[17] O. G. Miranda, D. K. Papoulias, M. Tórtola, and J. W. F. Valle, Probing neutrino transition magnetic moments with coherent elastic neutrino-nucleus scattering, J. High Energy Phys. 07 (2019) 103.

[18] A. Parada, New constraints on neutrino electric millicharge from elastic neutrino-electron scattering and coherent elastic neutrino-nucleus scattering, arXiv:1907.04942.

[19] T. S. Kosmas, D. K. Papoulias, M. Tortola, and J. W. F. Valle, Probing light sterile neutrino signatures at reactor and Spallation Neutron Source neutrino experiments, Phys. Rev. D 96, 063013 (2017).

[20] B. C. Cañas, E. A. Garcés, O. G. Miranda, and A. Parada, The reactor antineutrino anomaly and low energy threshold neutrino experiments, Phys. Lett. B 776, 451 (2018).

[21] C. Blanco, D. Hooper, and P. Machado, Constraining sterile neutrino interpretations of the LSND and MiniBooNE anomalies with coherent neutrino scattering experiments, Phys. Rev. D 101, 075051 (2020).

[22] D. Aristizabal Sierra, V. De Romeri, and N. Rojas, $C P$ violating effects in coherent elastic neutrino-nucleus scattering processes, J. High Energy Phys. 09 (2019) 069

[23] J. B. Dent, B. Dutta, S. Liao, J. L. Newstead, L. E. Strigari, and J. W. Walker, Probing light mediators at ultralow threshold energies with coherent elastic neutrino-nucleus scattering, Phys. Rev. D 96, 095007 (2017).

[24] Y. Farzan, M. Lindner, W. Rodejohann, and X.-J. Xu, Probing neutrino coupling to a light scalar with coherent neutrino scattering, J. High Energy Phys. 05 (2018) 066.

[25] M. Abdullah, J. B. Dent, B. Dutta, G. L. Kane, S. Liao, and L. E. Strigari, Coherent elastic neutrino nucleus scattering as a probe of a $Z^{\prime}$ through kinetic and mass mixing effects, Phys. Rev. D 98, 015005 (2018).

[26] V. Brdar, W. Rodejohann, and X.-J. Xu, Producing a new fermion in coherent elastic neutrino-nucleus scattering: From neutrino mass to dark matter, J. High Energy Phys. 12 (2018) 024.

[27] M. Cadeddu, C. Giunti, Y. F. Li, and Y. Y. Zhang, Average CsI Neutron Density Distribution from COHERENT Data, Phys. Rev. Lett. 120, 072501 (2018). 
[28] E. Ciuffoli, J. Evslin, Q. Fu, and J. Tang, Extracting nuclear form factors with coherent neutrino scattering, Phys. Rev. D 97, 113003 (2018).

[29] X.-R. Huang and L.-W. Chen, Neutron skin in CsI and lowenergy effective weak mixing angle from COHERENT data, Phys. Rev. D 100, 071301 (2019).

[30] D. Aristizabal Sierra, J. Liao, and D. Marfatia, Impact of form factor uncertainties on interpretations of coherent elastic neutrino-nucleus scattering data, J. High Energy Phys. 06 (2019) 141.

[31] D. K. Papoulias, T. S. Kosmas, R. Sahu, V. K. B. Kota, and M. Hota, Constraining nuclear physics parameters with current and future COHERENT data, Phys. Lett. B 800, 135133 (2020).

[32] G. Arcadi, M. Lindner, J. Martins, and F. S. Queiroz, New physics probes: Atomic parity violation, polarized electron scattering and neutrino-nucleus coherent scattering, Nucl. Phys. B959, 115158 (2020).

[33] M. Cadeddu, F. Dordei, C. Giunti, K. A. Kouzakov, E. Picciau, and A. I. Studenikin, Potentialities of a low-energy detector based on ${ }^{4} \mathrm{He}$ evaporation to observe atomic effects in coherent neutrino scattering and physics perspectives, Phys. Rev. D 100, 073014 (2019).

[34] D. K. Papoulias, R. Sahu, T. S. Kosmas, V. K. B. Kota, and B. Nayak, Novel neutrino-floor and dark matter searches with deformed shell model calculations, Adv. High Energy Phys. 2018, 6031362 (2018).

[35] C. Bœhm, D. G. Cerdeño, P. A. N. Machado, A. OlivaresDel Campo, E. Perdomo, and E. Reid, How high is the neutrino floor? J. Cosmol. Astropart. Phys. 01 (2019) 043.

[36] J. M. Link and X.-J. Xu, Searching for BSM neutrino interactions in dark matter detectors, J. High Energy Phys. 08 (2019) 004.

[37] S.-F. Ge and I. M. Shoemaker, Constraining photon portal dark matter with texono and coherent data, J. High Energy Phys. 11 (2018) 066.

[38] K. C. Y. Ng, J. F. Beacom, A. H. G. Peter, and C. Rott, Solar atmospheric neutrinos: A new neutrino floor for dark matter searches, Phys. Rev. D 96, 103006 (2017).

[39] D. Akimov et al. (COHERENT Collaboration), COHERENT 2018 at the Spallation Neutron Source, arXiv:1803 .09183 .

[40] J. Hakenmüller et al., Neutron-induced background in the CONUS experiment, Eur. Phys. J. C 79, 699 (2019).

[41] A. Aguilar-Arevalo et al. (CONNIE Collaboration), Results of the engineering run of the Coherent Neutrino Nucleus Interaction Experiment (CONNIE), J. Instrum. 11, P07024 (2016).

[42] G. Agnolet et al. (MINER Collaboration), Background studies for the MINER coherent neutrino scattering reactor experiment, Nucl. Instrum. Methods Phys. Res., Sect. A 853, 53 (2017).

[43] J. Billard et al., Coherent neutrino scattering with low temperature bolometers at Chooz reactor complex, J. Phys. G 44, 105101 (2017).

[44] R. Strauss et al., The $\nu$-cleus experiment: A gram-scale fiducial-volume cryogenic detector for the first detection of coherent neutrino-nucleus scattering, Eur. Phys. J. C 77, 506 (2017).
[45] H. T. Wong, Neutrino-nucleus coherent scattering and dark matter searches with sub-keV germanium detector, Nucl. Phys. A844, 229C (2010).

[46] V. Belov et al., The vGeN experiment at the Kalinin Nuclear Power Plant, J. Instrum. 10, P12011 (2015).

[47] D. Y. Akimov et al., RED-100 detector for the first observation of the elastic coherent neutrino scattering off xenon nuclei, J. Phys. Conf. Ser. 675, 012016 (2016).

[48] D. Yu. Akimov, V. A. Belov, A. Bolozdynya, Yu. V. Efremenko, A. M. Konovalov, A. V. Kumpan, D. G. Rudik, V. V. Sosnovtsev, A. V. Khromov, and A. V. Shakirov, Coherent elastic neutrino scattering on atomic nucleus: Recently discovered type of low-energy neutrino interaction, Usp. Fiz. Nauk 189, 173 (2019) [Phys. Usp. 62, 166 (2019)].

[49] J. I. Collar, N. E. Fields, M. Hai, T. W. Hossbach, J. L. Orrell, C. T. Overman, G. Perumpilly, and B. Scholz, Coherent neutrino-nucleus scattering detection with a CsI [Na] scintillator at the SNS spallation source, Nucl. Instrum. Methods Phys. Res., Sect. A 773, 56 (2015).

[50] J. I. Collar, Quenching and channeling of nuclear recoils in $\mathrm{NaI}(\mathrm{Tl})$ : Implications for dark-matter searches, Phys. Rev. C 88, 035806 (2013).

[51] B. J. Scholz, First observation of coherent elastic neutrinonucleus scattering, Ph.D. thesis, Chicago University, 2017.

[52] A. Konovalov, in Magnificent CEvNS (2020), https://indico .cern.ch/event/943069/contributions/4066385/.

[53] J. I. Collar, A. R. L. Kavner, and C. M. Lewis, Response of CsI[Na] to nuclear recoils: Impact on coherent elastic neutrino-nucleus scattering (CE $\nu \mathrm{NS})$, Phys. Rev. D 100, 033003 (2019).

[54] W. C. Louis, Searches for muon-to-electron (anti) neutrino flavor change, Prog. Part. Nucl. Phys. 63, 51 (2009).

[55] D. Z. Freedman, Coherent neutrino nucleus scattering as a probe of the weak neutral current, Phys. Rev. D 9, 1389 (1974).

[56] D. K. Papoulias and T. S. Kosmas, Standard and nonstandard neutrino-nucleus reactions cross sections and event rates to neutrino detection experiments, Adv. High Energy Phys. 2015, 763648 (2015).

[57] V. A. Bednyakov and D. V. Naumov, Coherency and incoherency in neutrino-nucleus elastic and inelastic scattering, Phys. Rev. D 98, 053004 (2018).

[58] J. Barranco, O. G. Miranda, and T. I. Rashba, Probing new physics with coherent neutrino scattering off nuclei, J. High Energy Phys. 12 (2005) 021.

[59] M. Tanabashi et al. (Particle Data Group Collaboration), Review of particle physics, Phys. Rev. D 98, 030001 (2018).

[60] S. Klein and J. Nystrand, Exclusive vector meson production in relativistic heavy ion collisions, Phys. Rev. C 60, 014903 (1999).

[61] COHERENT Collaboration (private communication).

[62] R. H. Helm, Inelastic and elastic scattering of 187-Mev electrons from selected even-even nuclei, Phys. Rev. 104, 1466 (1956).

[63] O. G. Miranda and H. Nunokawa, Non standard neutrino interactions: Current status and future prospects, New J. Phys. 17, 095002 (2015). 
[64] Y. Farzan and M. Tortola, Neutrino oscillations and nonstandard interactions, Front. Phys. 6, 10 (2018).

[65] J. Barranco, O. G. Miranda, C. A. Moura, and J. W. F. Valle, Constraining non-standard interactions in $\nu_{e} e$ or $\bar{\nu}_{e} e$ scattering, Phys. Rev. D 73, 113001 (2006).

[66] K. Scholberg, Prospects for measuring coherent neutrinonucleus elastic scattering at a stopped-pion neutrino source, Phys. Rev. D 73, 033005 (2006).

[67] J. Schechter and J. W. F. Valle, Majorana neutrinos and magnetic fields, Phys. Rev. D 24, 1883 (1981); Erratum, Phys. Rev. D 25, 283 (1982).

[68] W. Grimus and T. Schwetz, Elastic neutrino electron scattering of solar neutrinos and potential effects of magnetic and electric dipole moments, Nucl. Phys. B587, 45 (2000).

[69] M. Tortola, Constraining neutrino magnetic moment with solar and reactor neutrino data, Proc. Sci., AHEP2003 (2003) 022 [arXiv:hep-ph/0401135].

[70] B. C. Cañas, O. G. Miranda, A. Parada, M. Tortola, and J.W.F. Valle, Updating neutrino magnetic moment constraints, Phys. Lett. B 753, 191 (2016).

[71] P. Vogel and J. Engel, Neutrino electromagnetic form factors, Phys. Rev. D 39, 3378 (1989).
[72] C. Giunti and A. Studenikin, Neutrino electromagnetic interactions: A window to new physics, Rev. Mod. Phys. 87, 531 (2015).

[73] M. Hirsch, E. Nardi, and D. Restrepo, Bounds on the tau and muon neutrino vector and axial vector charge radius, Phys. Rev. D 67, 033005 (2003).

[74] M. Cadeddu, C. Giunti, K. A. Kouzakov, Y. F. Li, A. I. Studenikin, and Y. Y. Zhang, Neutrino charge radii from COHERENT elastic neutrino-nucleus scattering, Phys. Rev. D 98, 113010 (2018).

[75] M. Cadeddu, F. Dordei, C. Giunti, Y. F. Li, and Y. Y. Zhang, Neutrino, electroweak and nuclear physics from COHERENT elastic neutrino-nucleus scattering with refined quenching factor, Phys. Rev. D 101, 033004 (2020).

[76] E. Bertuzzo, F. F. Deppisch, S. Kulkarni, Y. F. Perez Gonzalez, and R. Zukanovich Funchal, Dark matter and exotic neutrino interactions in direct detection searches, J. High Energy Phys. 04 (2017) 073.

[77] D. G. Cerdeño, M. Fairbairn, T. Jubb, P. A. N. Machado, A. C. Vincent, and C. Bœhm, Physics from solar neutrinos in dark matter direct detection experiments, J. High Energy Phys. 05 (2016) 118; Erratum, J. High Energy Phys. 09 (2016) 048. 